515.537 .6

\author{
I. Volyanska, V. Il'Kiv, N. Strap
}

\title{
TWO-POINT NONLOCAL PROBLEM FOR A WEAK NONLINEAR DIFFERENTIAL-OPERATOR EQUATION
}

\begin{abstract}
I. Volyanska, V. Il'kiv, N. Strap. Two-point nonlocal problem for a weak nonlinear differentialoperator equation, Mat. Stud. 50 (2018), 44-59.

We study the solvability of a two-point nonlocal boundary-value problem for an operatordifferential equation with weakly nonlinear right-hand side. The proof of theorems is carried out within the Nash-Moser iterative scheme. In this scheme, the important point is a construction of estimates to norms of inverse linearized operators arising at each step of this scheme as well as the related problem of small denominators. The inverse operator for linearized operator is obtained by the method developed in the work of M. Berti, P. Bolle (Duke Math. J., 134 (2), 359-419 (2006)). The problem of small denominators is solved by using the metric approach.
\end{abstract}

1. Introduction. Problems with nonlocal conditions for partial differential equations represent an important part of the modern theory of differential equations $[11,12,15]$. Such problems are mainly ill posed in the Hadamard sence, and their solvability is connected with the problem of small denominators that arise in the construction of solutions $[1,5,6,15]$.

A specific feature of the present work is the study of a nonlocal boundary-value problem for an operator-differential equation with nonlinear right-hand side. The proof of the solvability of problem will be executed by the Nash-Moser iterative scheme [13,14].

Using the Nash-Moser iterative scheme Berti and Bolle [2] proved the existence of small amplitude, $2 \pi / \omega$-periodic in time solution of completely resonant nonlinears with Dirichlet boundary conditions, for any $\omega$ belonging to a Cantor-like set of positive measure. The same problem for the wave equations with nonlinearities of class $C^{k}$ has been investigated in [3] (required a modified Nash-Moser iterative scheme with interpolation estimates for the inverse linearized operators). In [4] it has been proved the existence of Cantor families of periodic solutions for nonlinear wave equations in higher spatial dimensions with periodic boundary conditions. The proofs are based on a differentiable Nash-Moser iterative scheme.

The nonlocal boundary value problems for a differential equation with nonlinearity and with operator $B=\left(B_{1}, \ldots, B_{p}\right)$, where $B_{j} \equiv z_{j} \frac{\partial}{\partial z_{j}}, j=1, \ldots, p$, were considered in the papers [7-10]. By using of the Nash-Mozer iteration scheme, conditions of the solvability of the problems in the Sobolev spaces were established for the functions of several complex variables, in the Hörmander-Hilbert spaces and in the scale of spaces of functions, which are Dirichlet-Taylor series with fixed spectrum.

In this work, the crucial point is a construction of estimates of norms of inverse linearized operators in each iteration. The estimation is related to the problem of small denominators, which is solved within metric approach.

2010 Mathematics Subject Classification: 35G15, 35E05.

Keywords: nonlocal problem; small denominators; metric estimation; Nash-Moser iterative scheme. doi:10.15330/ms.50.1.44-59

(C) I. Volyanska, V. Il'kiv, N. Strap, 2018 
2. Notations and statement of the problem. Let $\mathbf{X}$ be a separable Hilbert space; $A_{1}, \ldots, A_{p}$ be linear operators that have a common spectral representation, that is, there is a complete orthonormal system of elements $x_{k} \in \mathbf{X}, k \in \mathbb{N}$ such that the equalities

$$
A_{i} x_{k}=\alpha_{i k} x_{k}, \quad i=1, \ldots, p, \quad k \in \mathbb{N},
$$

hold for some complex numbers $\alpha_{i k}$.

Write $\alpha_{k}=\left(\alpha_{1 k}, \ldots, \alpha_{p k}\right),\left\|\alpha_{k}\right\|^{2}=\left|\alpha_{1 k}\right|^{2}+\ldots+\left|\alpha_{p k}\right|^{2}$, and let

$$
\left\|\alpha_{k}\right\|>C k^{\beta_{0}}, \quad C>0, \quad \beta_{0}>0 .
$$

We introduce the function $\zeta_{1}(x)=\sum_{k \in \mathbb{N}}\left(1+\left\|\alpha_{k}\right\|^{2}\right)^{-x}$ defined for $x>1 /\left(2 \beta_{0}\right)$, and also the function $\zeta_{2}(x)=\sum_{m \in \mathbb{Z}}\left(1+|m|^{2}\right)^{-x}$ defined for $x>1 / 2$.

Denote $\tau(m)=-\ln \mu / T+i 2 \pi m / T$, where $\ln \mu$ is the principal value of the logarithm. Let us consider the spaces $\mathbf{X}_{d, r}(\Omega), d, r \in \mathbb{R}, \Omega \subseteq(\mathbb{N} \times \mathbb{Z})$, being the Hilbert spaces of functions

$$
u(t, \Omega)=\sum_{(k, m) \in \Omega} u_{k, m} e^{\tau(m) t} x_{k},
$$

equipped with the scalar product

$$
(u(\cdot, \Omega), v(\cdot, \Omega))_{d, r, \Omega}=\sum_{(k, m) \in \Omega}\left(1+\left\|\alpha_{k}\right\|^{2}\right)^{d}\left(1+m^{2}\right)^{r} u_{k, m} \bar{v}_{k, m}
$$

where

$$
v(t, \Omega)=\sum_{(k, m) \in \Omega} v_{k, m} e^{\tau(m) t} x_{k}
$$

$\bar{v}_{k, m}$ is complex conjugate with $v_{k, m}$.

The scalar product induces the norm $\|\cdot\|_{d, r, \Omega}$. For simplicity, we denote $\mathbf{X}_{d, r}(\mathbb{N} \times \mathbb{Z})=\mathbf{X}_{d, r}$, $u(t, \mathbb{N} \times \mathbb{Z})=u(t),\|\cdot\|_{d, r, \mathbb{N} \times \mathbb{Z}}=\|\cdot\|_{d, r}$.

Note if $u \in \mathbf{X}_{d, r}$, then $\|u\|_{d, r, \Omega} \leq\|u\|_{d, r}$ for any $d, r$ and $\Omega$.

Obviously, if $\Omega=\Omega_{1} \cup \Omega_{2}$ and $\Omega_{1} \cap \Omega_{2}=\varnothing$, then $\mathbf{X}_{d, r}(\Omega)=\mathbf{X}_{d, r}\left(\Omega_{1}\right) \oplus \mathbf{X}_{d, r}\left(\Omega_{2}\right)$, where $\oplus$ means the direct sum; so for any function $u(t, \Omega) \in \mathbf{X}_{d, r}(\Omega) u(t, \Omega)=u\left(t, \Omega_{1}\right)+u\left(t, \Omega_{2}\right)$ holds, where $u\left(t, \Omega_{1}\right) \in \mathbf{X}_{d, r}\left(\Omega_{1}\right), u\left(t, \Omega_{2}\right) \in \mathbf{X}_{d, r}\left(\Omega_{2}\right)$.

The operators $\frac{d}{d t}: \mathbf{X}_{d, r}(\Omega) \rightarrow \mathbf{X}_{d, r-1}(\Omega)$ and $A_{j}: \mathbf{X}_{d, r}(\Omega) \rightarrow \mathbf{X}_{d-1, r}(\Omega), j=1, \ldots, p$, act as follows

$$
\frac{d u}{d t}=\sum_{(k, m) \in \Omega} \tau(m) u_{k, m} e^{\tau(m) t} x_{k}, \quad A_{j} u=\sum_{(k, m) \in \Omega} \alpha_{j k} u_{k, m} e^{\tau(m) t} x_{k}
$$

for any

$$
u=u(t, \Omega)=\sum_{(k, m) \in \Omega} u_{k, m} e^{\tau(m) t} x_{k} \in \mathbf{X}_{d, r}
$$

and $(d, r) \in \mathbb{R}^{2}$.

Let us consider the problem with nonlocal conditions for the operator-differential equation with constant coefficients and a nonlinear right-hand side

$$
L\left(d_{t}, A\right) u \equiv \sum_{|\hat{s}| \leq n} a_{\hat{s}} A_{1}^{s_{1}} \ldots A_{p}^{s_{p}} d_{t}^{s_{0}} u(t)=\varepsilon f(u), \quad d_{t}=d / d t
$$




$$
\left.\mu d_{t}^{m} u\right|_{t=0}-\left.d_{t}^{m} u\right|_{t=T}=0, \quad m=0,1, \ldots, n-1,
$$

in the space $\mathbf{X}_{d, r}$, where $a_{\hat{s}}, \varepsilon$ and $\mu$ are complex parameters $\left(a_{(n, 0, \ldots, 0)}=1, \mu \neq 0\right)$, and $\hat{s}=\left(s_{0}, s\right), s=\left(s_{1}, \ldots, s_{p}\right) \in \mathbb{Z}_{+}^{p},|\hat{s}|=s_{0}+s_{1}+\ldots+s_{p}$.

Let us consider the eigenvalue problem for the operator $\mathrm{L}$ in $\mathbf{X}_{d, r}$, generated by the differential expression $L\left(d_{t}, A\right)$ and the boundary conditions $(2)$ with $\mu \neq 0$ :

$$
L\left(d_{t}, A\right) u=\lambda u,\left.\quad \mu d_{t}^{m} u\right|_{t=0}-\left.d_{t}^{m} u\right|_{t=T}=0, \quad m=0,1, \ldots, n-1,
$$

where $\lambda$ is a spectral parameter.

Given vector $(k, m) \in \mathbb{N} \times \mathbb{Z}$ we denote by $R_{k, m}$ the set of vectors $\left(k^{*}, m^{*}\right) \in \mathbb{N} \times \mathbb{Z}$, for which the equality $L\left(\tau\left(m^{*}\right), \alpha_{k^{*}}\right)=L\left(\tau(m), \alpha_{k}\right)$ holds, where $\tau(m)=-\ln \mu / T+i 2 \pi m / T$.

The eigenvalues of problem (3) are the numbers $\lambda_{k, m}=L\left(\tau(m), \alpha_{k}\right),(k, m) \in \mathbb{N} \times \mathbb{Z}$, the eigenfunctions, corresponding to the eigenvalue $\lambda_{k, m}$, are the functions $x_{k^{*}} e^{\tau\left(m^{*}\right) t},\left(k^{*}, m^{*}\right) \in$ $R_{k, m}[15]$.

We look for a solution of problem (1), (2) by using the Nash-Moser iteration scheme in the form of the limit of a some sequence of smooth functions.

For any $N \in \mathbb{N}$ we represent the space $\mathbf{X}_{d, r}$ as the direct sum $\mathbf{X}_{d, r}=\mathbf{W}^{(N)} \oplus \mathbf{W}^{(N) \perp}$, where

$$
\begin{gathered}
\mathbf{W}^{(N)}=\left\{u \in \mathbf{X}_{d, r}: u=\sum_{(k, m) \in \Omega^{N}} u_{k, m} e^{\tau(m) t} x_{k}\right\}, \Omega^{N}=\left\{(k, m): 1+\left\|\alpha_{k}\right\|^{2} \leq N, 1+m^{2} \leq N\right\}, \\
\mathbf{W}^{(N) \perp}=\left\{u \in \mathbf{X}_{d, r}: u=\sum_{(k, m) \in(\mathbb{N} \times \mathbb{Z}) \backslash \Omega^{N}} u_{k, m} e^{\tau(m) t} x_{k}\right\} .
\end{gathered}
$$

We denote by $\mathrm{P}_{N}: \mathbf{X}_{d, r} \rightarrow \mathbf{W}^{(N)}$ and $\mathrm{P}_{N}^{\perp}: \mathbf{X}_{d, r} \rightarrow \mathbf{W}^{(N) \perp}, N \in \mathbb{N}$, the projection operators in the space $\mathbf{X}_{d, r}$ onto $\mathbf{W}^{(N)}$ and $\mathbf{W}^{(N) \perp}$, respectively, i.e., $\mathbf{W}^{(N)}=\mathbf{P}_{N} \mathbf{X}_{d, r}$, $\mathbf{W}^{(N) \perp}=\mathrm{P}_{N}^{\perp} \mathbf{X}_{d, r}$. For any function $u \in \mathbf{X}_{d, r}$ they are defined by the following formulas:

$$
\mathrm{P}_{N} u=\sum_{(k, m) \in \Omega^{N}} u_{k, m} e^{\tau(m) t} x_{k}, \quad \mathrm{P}_{N}^{\perp} u=\sum_{(k, m) \in(\mathbb{N} \times \mathbb{Z}) \backslash \Omega^{N}} u_{k, m} e^{\tau(m) t} x_{k} .
$$

The definitions of the space $\mathbf{X}_{d, r}$ and the projector $\mathrm{P}_{N}$ imply that, for any $N \in \mathbb{N}, d \in \mathbb{R}$, $r \in \mathbb{R}$ the following inequalities hold:

$$
\begin{gathered}
\left\|\mathrm{P}_{N} u\right\|_{d+j_{1}, r+j_{2}} \leq N^{j_{1}+j_{2}}\|u\|_{d, r} \quad \text { for every } \quad u \in \mathbf{X}_{d, r}, \\
\left\|\mathrm{P}_{N}^{\perp} u\right\|_{d, r} \leq N^{-j_{1}-j_{2}}\|u\|_{d+j_{1}, r+j_{2}} \quad \text { for every } \quad u \in \mathbf{X}_{d+j_{1}, r+j_{2}} .
\end{gathered}
$$

The existence of a solution of problem (1), (2) is based on properties $(P 1)-(P 5)$ of the coefficients $a_{\hat{s}}$ and the function $f$ on the right-hand side of the equation, which maps, by assumption, the space $\mathbf{X}_{d, r}$ into itself.

Write $\Omega_{1}=\left\{(k, m) \in \mathbb{N} \times \mathbb{Z}:|\tau(m)|<\left\|\alpha_{k}\right\|\right\}, \Omega_{2}=(\mathbb{N} \times \mathbb{Z}) \backslash \Omega_{1}$.

Let $l \geq d+2, m \geq r+2, C_{0} \geq 0, C_{1} \geq 0$ and $C_{2} \geq 0$ be such that conditions $(P 1)-(P 4)$ are satisfied:

(P1) $f \in \mathbf{C}^{2}\left(\mathbf{X}_{d, r} ; \mathbf{X}_{d, r}\right)$, in particular, $f, \mathrm{D}_{u} f, \mathrm{D}_{u}^{2} f$ are bounded on the ball

$$
K_{1}=\left\{u \in \mathbf{X}_{d, r}:\|u\|_{d, r} \leq 1\right\} \text { from the space } \mathbf{X}_{d, r} .
$$

(P2) For any $d^{\prime} \in[d, l), r^{\prime} \in[r, m)$ and function $u \in \mathbf{X}_{d^{\prime}, r^{\prime}}$, the inequality

$$
\|f(u)\|_{d^{\prime}, r^{\prime}} \leq C_{0}\left(1+\|u\|_{d^{\prime}, r^{\prime}}\right) \text { holds. }
$$


(P3) For any $u \in K_{1}$ and $h \in \mathbf{X}_{d, r}$ there exists $\overline{\bar{d}}>d+2 \delta, \overline{\bar{r}}>r+2 \delta$, where $\delta=\kappa+\alpha-$ $n / 2, \kappa \geq 1 /\left(4 \beta_{0}\right), \alpha>1 / 4$, such that $D_{u} f(u) \in \mathbf{C}^{1}\left(\mathbf{X}_{d, r} ; \mathbf{X}_{\overline{\bar{d}}, \overline{\bar{r}}}\right)$ and $\left\|\mathrm{D}_{u} f(u)[h]\right\|_{\overline{\bar{d}}, \overline{\bar{r}}} \leq$ $C_{1}\|h\|_{d, r}$.

(P4) For any $d^{\prime} \in[d, l-2], r^{\prime} \in[r, m-2], u \in \mathbf{X}_{d^{\prime}, r^{\prime}} \cap K_{1}$ and $h \in \mathbf{X}_{d^{\prime}, r^{\prime}}$ the inequality

$$
\left\|f(u+h)-f(u)-\mathrm{D}_{u} f(u) h\right\|_{d^{\prime}, r^{\prime}} \leq C_{2}\left(\|u\|_{d^{\prime}, r^{\prime}}\|h\|_{d, r}^{2}+\|h\|_{d, r}\|h\|_{d^{\prime}, r^{\prime}}\right)
$$

holds.

Property $(P 4)$ yields

$$
\left\|f(u+h)-f(u)-\mathrm{D}_{u} f(u) h\right\|_{d, r} \leq 2 C_{2}\|h\|_{d, r}^{2}, \quad u \in K_{1}, \quad h \in \mathbf{X}_{d, r} .
$$

Here is an example of a function $f(u)$ that satisfies the conditions $(P 1)-(P 4)$. For any element $u=\sum_{(k, m) \in \mathbb{N} \times \mathbb{Z}} u_{k, m} e^{\tau(m) t} x_{k}$ from the $\mathbf{X}_{d, r}$ the element $f(u)$ is given by

$$
f(u)=\sum_{k+|m| \leq Q}\left(f_{k, m} u_{k, m}\right)^{\sigma} e^{\tau(m) t} x_{k}
$$

where $Q$ is a natural number, $f_{k, m}$ are arbitrary complex numbers, $\sigma>2$.

This four properties characterize the behavior of a function $f$ in the ball $K_{1}$ of the space $\mathbf{X}_{d, r}$. In order to formulate property $(P 5)$ characterizing the coefficients $a_{\hat{s}}$ of equation (1), we assume that they belong to the disk $\mathcal{O}_{A}=\{z \in \mathbb{C}:|z|<A\}$.

Let us introduce the vectors

$$
\vec{\varepsilon}=(\operatorname{Re} \varepsilon, \operatorname{Im} \varepsilon), \quad \vec{a}=\left(\operatorname{Re} a_{\hat{s}(j)}, \operatorname{Im} a_{\hat{s}(j)}\right)_{j=0,1, \ldots, p}, \hat{s}(j)=(\underbrace{0, \ldots, 0}_{j}, n, 0, \ldots, 0) \in \mathbb{Z}_{+}^{p} .
$$

We now introduce a sequence of integer numbers

$$
N_{q}=N_{0}^{2^{q}}\left(=N_{q-1}^{2}\right)
$$

with some integer number $N_{0} \geq 2$.

We consider the operator L on the set of parameters $\vec{a} \in \mathcal{O}_{A}^{p+1}$, and all other $a_{\hat{s}}$ to be fixed. In view of conditions $(P 3)$ for $\gamma>0$ we can construct the sequence of sets $G_{0}, G_{1}, \ldots$, where $G_{q}, q=0,1, \ldots$, is the set of those vectors $\vec{a}$ from equation (1), for which the following estimate holds:

$$
\left|\lambda_{k, m}\right|>\gamma\left(1+\left\|\alpha_{k}\right\|^{2}\right)^{-\kappa_{j}}\left(1+m^{2}\right)^{-\alpha_{j}} \quad \text { for } \quad(k, m) \in \Omega^{N_{q}} \cap \Omega_{j}, \quad j=1,2,
$$

where $\kappa_{j}=\kappa-n \delta_{1 j} / 2, \alpha_{j}=\alpha-n \delta_{2 j} / 2$, and $\delta_{i j}$ is the Kronecker delta.

The definition of the sets $G_{q}$ yields the embeddings $\mathcal{O}_{A}^{p+1} \supset G_{0} \supseteq G_{1} \supseteq \ldots$

Introduce the sets $\mathcal{G}_{q}$ corresponding to the sets $G_{q}$ by the formula $\mathcal{G}_{q}=\mathcal{O}_{\varepsilon_{0}} \times G_{q}$, where $q \geq 0$,

$$
\varepsilon_{0}=\gamma \min \left\{\frac{3}{16 C_{3}}, \frac{1}{2 C_{0} N_{0}^{2 \delta}}, y_{1}, y_{2}\right\}, \quad C_{3}=\max \left\{C_{0}, C_{1}, 2 C_{2}\right\}
$$

$y_{1}, y_{2}$ are positive solutions of the equations

$$
2 C_{0} N_{0}^{14 \delta} y^{3}+y^{2}=\frac{1}{24} C_{3}^{-2}, \quad 2 C_{0} N_{0}^{6 \delta} y^{2}+\left(2+N_{0}^{-8 \delta}\right) y=\frac{3}{4} C_{3}^{-1}
$$


respectively. Then the embeddings

$$
\ldots \subseteq \mathcal{G}_{1} \subseteq \mathcal{G}_{0} \subset \mathcal{O}_{\varepsilon_{0}} \times \mathcal{O}_{A}^{p+1}
$$

are obvious.

For any functions $u \in \mathbf{W}^{(N)}, h \in \mathbf{W}^{(N)}$, and the parameter $\varepsilon \in \mathbb{C} \backslash\{0\}$, we set

$$
\mathcal{L}_{N}[h] \equiv \mathcal{L}_{N}(\vec{\varepsilon}, \vec{a}, u)[h]=L h-\varepsilon \mathrm{P}_{N} \mathrm{D}_{u} f(u) h, \quad N \in \mathbb{N}
$$

where $L$ stands on the left-hand side of equation (1), and the projector $\mathrm{P}_{N}$ is defined by formula (4).

The following property $(P 5)$ concerns the continuity of the inverse operator for the linear operator $\mathcal{L}_{N_{q}}(\vec{\varepsilon}, \vec{a}, u): \mathbf{W}^{\left(N_{q}\right)} \rightarrow \mathbf{W}^{\left(N_{q}\right)}$, where $q \geq 0$ :

(P5) For any $u \in \mathbf{W}^{\left(N_{q}\right)} \cap K_{1}$ and $\gamma>0$ for all vectors $(\vec{\varepsilon}, \vec{a}) \in \mathcal{G}_{q}$ operator

$\mathcal{L}_{N_{q}}(\vec{\varepsilon}, \vec{a}, u): \mathbf{W}^{\left(N_{q}\right)} \rightarrow \mathbf{W}^{\left(N_{q}\right)}$ is invertible; in particular, for $\bar{d} \in[d, \overline{\bar{d}}-2 \delta], \bar{r} \in[r, \overline{\bar{r}}-2 \delta]$ and $h \in \mathbf{W}^{\left(N_{q}\right)}$ the estimate

$$
\left\|\mathcal{L}_{N_{q}}^{-1}(\varepsilon, \vec{a}, u)[h]\right\|_{\bar{d}, \bar{r}} \leq \frac{2}{\gamma} N_{q}^{2 \delta}\|h\|_{\bar{d}, \bar{r}}, \quad q \in \mathbb{N}
$$

holds.

Proof of property (P5). Given $q \geq 0$; we represent the operator $\mathcal{L}_{N_{q}}$ in the form $\mathcal{L}_{N_{q}}=$ $\mathcal{D}-\mathcal{T}_{q}$, where $\mathcal{D}$ is a diagonal operator, $\mathcal{D}=L, \mathcal{T}_{q}=\varepsilon \mathrm{P}_{N_{q}} \mathrm{D}_{u} f$. Then we have

$$
\mathcal{L}_{N_{q}}=|\mathcal{D}|^{1 / 2} \mathcal{U}|\mathcal{D}|^{1 / 2}-\mathcal{T}_{q}=|\mathcal{D}|^{1 / 2}\left(\mathcal{U}-\mathcal{R}_{1}\right)|\mathcal{D}|^{1 / 2}=|\mathcal{D}|^{1 / 2} \mathcal{U}\left(\mathcal{I}-\mathcal{U}^{-1} \mathcal{R}_{1}\right)|\mathcal{D}|^{1 / 2},
$$
where $\mathcal{U}=|\mathcal{D}|^{-1 / 2} \mathcal{D}|\mathcal{D}|^{-1 / 2}, \mathcal{R}_{1}=|\mathcal{D}|^{-1 / 2} \mathcal{T}_{q}|\mathcal{D}|^{-1 / 2}$.

Suppose that

$$
h=\sum_{(k, m) \in \mathbb{N} \times \mathbb{Z}} h_{k, m} \varphi_{k, m}
$$

then diagonal operators $\mathcal{D}$ and $|\mathcal{D}|^{\nu}, \nu \geq 0$, in the space $\left\{\mathbf{X}_{d, r}\right\}_{d, r \in \mathbb{R}}$ are represented by the formulas

$$
\mathcal{D} h=\sum_{(k, m) \in \mathbb{N} \times \mathbb{Z}} \lambda_{k, m} h_{k, m} \varphi_{k, m}, \quad|\mathcal{D}|^{\nu} h=\sum_{(k, m) \in \mathbb{N} \times \mathbb{Z}}\left|\lambda_{k, m}\right|^{\nu} h_{k, m} \varphi_{k, m} .
$$

For $\nu<0$ operators $|\mathcal{D}|^{\nu}$ exist if the condition $\lambda_{k, m} \neq 0$ holds for any $(k, m) \in \mathbb{N} \times \mathbb{Z}$. Operators $\mathcal{D}$ and $|\mathcal{D}|^{\nu}, \nu \in \mathbb{R}$, in the space $\mathbf{W}^{\left(N_{q}\right)}$ are represented by diagonal matrices and have eigenvalues $\lambda_{k, m}$ and $\left|\lambda_{k, m}\right|^{\nu}$, respectively, and eigenfunctions $\varphi_{k, m}=e^{\tau(m) t} x_{k}$ for $(k, m) \in \Omega^{N_{q}}$.

Lemma 1. For all vectors $\vec{a} \in G_{q}, q \geq 0$, the operator $|\mathcal{D}|$ is invertible in the space $\mathbf{W}^{\left(N_{q}\right)}$ and for any $d^{*}, r^{*} \in \mathbb{R}$ and $h \in \mathbf{W}^{\left(N_{q}\right)}$ the relation

is valid.

$$
\left\||\mathcal{D}|^{-1 / 2} h\right\|_{d^{*}, r^{*}, \Omega_{j}} \leq \frac{1}{\sqrt{\gamma}}\|h\|_{d^{*}+\kappa_{j}, r^{*}+\alpha_{j}, \Omega_{j}}, \quad j=1,2,
$$

The proof of this lemma is given in Section 4 .

For the operator $\mathcal{L}_{N_{q}}^{-1}$ we have a factorization similar to the factorization of $\mathcal{L}_{N_{q}}$ :

$$
\mathcal{L}_{N_{q}}^{-1}=|\mathcal{D}|^{-1 / 2}\left(\mathcal{I}-\mathcal{U}^{-1} \mathcal{R}_{1}\right)^{-1} \mathcal{U}^{-1}|\mathcal{D}|^{-1 / 2}=|\mathcal{D}|^{-1 / 2}(\mathcal{I}-\mathcal{R})^{-1} \mathcal{U}^{-1}|\mathcal{D}|^{-1 / 2}
$$


where $\mathcal{R}=\mathcal{U}^{-1} \mathcal{R}_{1}$ and $(\mathcal{I}-\mathcal{R})^{-1}=\mathcal{I}+\sum_{r=1}^{\infty} \mathcal{R}^{r}$

The last presentation is valid under the assumption of convergence of some geometric progression.

Lemma 2. Assume that the point spectrum of the operator $L$ does not contain zero. Then, for any $d^{*}, r^{*} \in \mathbb{R}$, the operator $\mathcal{U}$ is isometric in the space $\mathbf{X}_{d^{*}, r^{*}}$.

Lemma 3. For the operator $\mathcal{R}_{1}: \mathbf{W}^{\left(N_{q}\right)} \rightarrow \mathbf{W}^{\left(N_{q}\right)}, q \geq 0$, for all $\bar{d} \in[d, \overline{\bar{d}}-2 \delta], \bar{r} \in[r, \overline{\bar{r}}-2 \delta]$ and $u \in \mathbf{W}^{\left(N_{q}\right)}$ the estimate

holds.

$$
\left\|\mathcal{R}_{1} h\right\|_{\bar{d}, \bar{r}} \leq C_{1} \frac{|\varepsilon|}{\gamma}\|h\|_{\bar{d}, \bar{r}}
$$

The proofs of Lemmas 2 and 3 are given in Section 4.

The formula $\mathcal{R}=\mathcal{U}^{-1} \mathcal{R}_{1}$ and Lemmas 2 and 3 for all $\bar{d} \in[d, \overline{\bar{d}}-2 \delta], \bar{r} \in[r, \overline{\bar{r}}-2 \delta]$ yield

$$
\|\mathcal{R} h\|_{\bar{d}, \bar{r}}=\left\|\mathcal{U}^{-1} \mathcal{R}_{1} h\right\|_{\bar{d}, \bar{r}}=\left\|\mathcal{R}_{1} h\right\|_{\bar{d}, \bar{r}} \leq C_{1} \frac{|\varepsilon|}{\gamma}\|h\|_{\bar{d}, \bar{r}} .
$$

We have the estimate $\left\|(I-\mathcal{R})^{-1} h\right\|_{\bar{d}, \bar{r}} \leq\|h\|_{\bar{d}, \bar{r}}+\sum_{r \in \mathbb{N}}\left\|\mathcal{R}^{r} h\right\|_{\bar{d}, \bar{r}}$, where

$$
\left\|\mathcal{R}^{r} h\right\|_{\bar{d}, \bar{r}}=\left\|\mathcal{R}\left(\mathcal{R}^{r-1} h\right)\right\|_{\bar{d}, \bar{r}} \leq C_{1} \frac{|\varepsilon|}{\gamma}\left\|\mathcal{R}^{r-1} h\right\|_{\bar{d}, \bar{r}} \leq\left(C_{1} \frac{|\varepsilon|}{\gamma}\right)^{r}\|h\|_{\bar{d}, \bar{r}}
$$

Under condition $|\varepsilon|<\varepsilon_{0}$ and (10), we have $|\varepsilon|<3 \gamma / 16 C_{3}<\gamma / C_{1}$, and

$$
\left\|(\mathcal{I}-\mathcal{R})^{-1} h\right\|_{\bar{d}, \bar{r}} \leq\|h\|_{\bar{d}, \bar{r}} \sum_{r=0}^{\infty}\left(\frac{C_{1}|\varepsilon|}{\gamma}\right)^{r}=\|h\|_{\bar{d}, \bar{r}}\left(1-\frac{C_{1}|\varepsilon|}{\gamma}\right)^{-1}=\frac{\gamma}{\gamma-C_{1}|\varepsilon|}\|h\|_{\bar{d}, \bar{r}} .
$$

Returning to the estimate of the norm of operator $\mathcal{L}_{N_{q}}^{-1}$, from Lemmas 2 and 3 and formulas (6) and (10) we can show that, for any $\bar{d} \in[d, \overline{\bar{d}}-2 \delta], \bar{r} \in[r, \overline{\bar{r}}-2 \delta]$ and vectors $(\vec{\varepsilon}, \vec{a}) \in \mathcal{G}_{q}$, the following estimates hold:

$$
\begin{aligned}
\left\|\mathcal{L}_{N_{q}}^{-1} h\right\|_{\bar{d}, \bar{r}, \Omega_{j}} & \leq \frac{1}{\sqrt{\gamma}} \frac{\gamma}{\gamma-C_{1}|\varepsilon|}\left\||\mathcal{D}|^{-1 / 2} h\right\|_{\bar{d}+\kappa_{j}, \bar{r}+\alpha_{j}, \Omega_{j}} \leq \frac{1}{\gamma} \frac{\gamma}{\gamma-C_{1}|\varepsilon|}\|h\|_{\bar{d}+2 \kappa_{j}, \bar{r}+2 \alpha_{j}, \Omega_{j}} \leq \\
\leq & \frac{N_{q}^{2 \delta}}{\gamma-C_{1}|\varepsilon|}\|h\|_{\bar{d}, \bar{r}}=\frac{N_{q}^{2 \delta}}{\gamma / 2-C_{1}|\varepsilon|+\gamma / 2}\|h\|_{\bar{d}, \bar{r}} \leq \frac{2}{\gamma} N_{q}^{2 \delta}\|h\|_{\bar{d}, \bar{r} .} .
\end{aligned}
$$

So, $\left\|\mathcal{L}_{N_{q}}^{-1} h\right\|_{\bar{d}, \bar{r}} \leq \frac{2}{\gamma} N_{q}^{2 \delta}\|h\|_{\bar{d}, \bar{r}}$, and property (P5) is proved.

Inequality (11) holds for the vector $(\vec{\varepsilon}, \vec{a}) \in \mathcal{G}_{q}$. For any $\gamma>0$ the limit of the sequence of sets $\left\{\mathcal{G}_{q}\right\}_{q=0,1, \ldots}$ coincides with the set $\mathcal{G}_{\infty}=\mathcal{G}_{\infty}(\gamma)=\lim _{q \rightarrow \infty} \mathcal{G}_{q}$. On this set there exists a solution to problem (1), (2).

3. Establishment of the solvability conditions for problem (1), (2). Let us define a sequence $\left\{u_{q}\right\}_{q \geq 0}$ recurrently, where $u_{q} \in \mathbf{W}^{\left(N_{q}\right)}$ defined on $\mathcal{G}_{q}$. This sequence converges to solution $u \in \mathbf{X}_{d, r}$ of $(1),(2)$ for all vectors $(\vec{\varepsilon}, \vec{a}) \in \mathcal{G}_{\infty}$. We will also prove that the set $\mathcal{G}_{\infty}$ in the set $\mathcal{O}_{\varepsilon_{0}} \times \mathcal{O}_{A}^{p+1}$ of parameters of the problem (1), (2) is large enough and find the lower bound for its measure. 
Theorem 1. Let properties $(P 1)-(P 5)$ be valid, and let $\beta=6 \delta, \delta=\kappa+\alpha-n / 2$. Then there exists a sequence $\left\{u_{q}\right\}_{q \geq 0}$, where $u_{q}=\sum_{i=0}^{q} h_{i} \in \mathbf{W}^{\left(N_{q}\right)}$ is a solution of the equation

$$
L u_{q}-\varepsilon \mathrm{P}_{N_{q}} f\left(u_{q}\right)=0,
$$

which is defined for $(\vec{\varepsilon}, \vec{a}) \in \mathcal{G}_{q}$. Moreover, if $B_{q}=1+\left\|u_{q}\right\|_{d+\beta, r+\beta}$ for $q \in\{0\} \cup \mathbb{N}$, then $B_{0} \leq 1+\frac{|\varepsilon|}{\gamma} 2 C_{0} N_{0}^{14 \delta}, B_{q} \leq B_{0} N_{q+1}^{2 \delta}$ for $q \in \mathbb{N}$, and $\left\|h_{i}\right\|_{d, r} \leq 4 C_{3} B_{0} \frac{|\varepsilon|}{\gamma} N_{i}^{-2 \delta}$ for $i \in \mathbb{N}$.

Proof. We use the method of mathematical induction. In view of condition $(\vec{\varepsilon}, \vec{a}) \in \mathcal{G}_{0}$ we will find a solution of the equation

$$
L u-\varepsilon \mathrm{P}_{N_{0}} f(u)=0 .
$$

There exists an operator $L^{-1}: \mathbf{W}^{\left(N_{0}\right)} \rightarrow \mathbf{W}^{\left(N_{0}\right)}$ such that

$$
L^{-1} w=\sum_{(k, m) \in \Omega^{N_{0}}} \lambda_{k, m}^{-1} w_{k, m} \varphi_{k, m}=\sum_{(k, m) \in \Omega^{N_{0}}} \frac{w_{k, m} \varphi_{k, m}}{\lambda_{k, m}}
$$

for any $w=\sum_{(k, m) \in \Omega^{N_{0}}} w_{k, m} \varphi_{k, m}$. Then estimate (9) yields the inequality

$$
\begin{gathered}
\left\|L^{-1} w\right\|_{d, r, \Omega_{j}}^{2}=\sum_{(k, m) \in \Omega^{N_{0} \cap \Omega_{j}}}\left(1+\left\|\alpha_{k}\right\|^{2}\right)^{d}\left(1+m^{2}\right)^{r} \frac{\left|w_{k, m}\right|^{2}}{\left|\lambda_{k, m}\right|^{2}} \leq \\
\leq \sum_{(k, m) \in \Omega^{N_{0} \cap \Omega_{j}}} \frac{1}{\gamma^{2}}\left(1+\left\|\alpha_{k}\right\|^{2}\right)^{d+2 \kappa_{j}}\left(1+m^{2}\right)^{r+2 \alpha_{j}}\left|w_{k, m}\right|^{2}=\frac{1}{\gamma^{2}}\|w\|_{d+2 \kappa_{j}, r+2 \alpha_{j}, \Omega_{j}}^{2},
\end{gathered}
$$

$j=1,2$. We can reduce equation $\left(P_{N_{0}}\right)$ to the form $u=\varepsilon L^{-1} \mathrm{P}_{N_{0}} f(u)$ the following estimates hold

$$
\left\|L^{-1} w\right\|_{d, r, \Omega_{j}} \leq \frac{1}{\gamma}\|w\|_{d+2 \kappa_{j}, r+2 \alpha_{j}, \Omega_{j}} \leq \frac{1}{\gamma} N_{0}^{2 \delta}\|w\|_{d, r, \Omega_{j}} \leq \frac{1}{\gamma} N_{0}^{2 \delta}\|w\|_{d, r}, \quad j=1,2
$$

for $w \in \mathbf{W}^{\left(N_{0}\right)}$.

So, we have that $\left\|L^{-1} w\right\|_{d, r} \leq \frac{1}{\gamma} N_{0}^{2 \delta}\|w\|_{d, r}$.

Remark. If $C_{1}=0$, then $f(u)=f(0)$ and $u=\varepsilon L^{-1} \mathrm{P}_{N_{0}} f(0)$ is a unique solution of equation $\left(P_{N_{0}}\right)$ in the space $\mathbf{W}^{\left(N_{0}\right)}$ for all vectors $(\vec{\varepsilon}, \vec{a}) \in \mathcal{G}_{0}$.

We denote by $H_{0}: \mathbf{W}^{\left(N_{0}\right)} \rightarrow \mathbf{W}^{\left(N_{0}\right)}$ the operator defined $H_{0}(u)=\varepsilon L^{-1} \mathrm{P}_{N_{0}} f(u)$ on element $u \in \mathbf{W}^{\left(N_{0}\right)}$. In order to construct a solution of $\left(P_{N_{0}}\right)$ we must find a fixed point $u \in \mathbf{W}^{\left(N_{0}\right)}$ of the operator.

We will show that for every vector $(\vec{\varepsilon}, \vec{a}) \in \mathcal{G}_{0}$ the operator $H_{0}$ is a contraction mapping in the domain

$$
G_{0}=\left\{u \in \mathbf{W}^{\left(N_{0}\right)}:\|u\|_{d, r} \leq \rho_{0}=\frac{|\varepsilon|}{\gamma} 2 C_{0} N_{0}^{2 \delta}\right\} .
$$

Using inequality (12), property (P2), formulas (10) and inequality $|\varepsilon|<\varepsilon_{0}$, we get the estimate

$$
\left\|H_{0}(u)\right\|_{d, r} \leq \frac{|\varepsilon|}{\gamma} N_{0}^{2 \delta}\|f(u)\|_{d, r} \leq \frac{|\varepsilon|}{\gamma} N_{0}^{2 \delta} C_{0}\left(1+\|u\|_{d, r}\right) \leq \frac{|\varepsilon|}{\gamma} N_{0}^{2 \delta} C_{0}\left(1+\rho_{0}\right)=
$$




$$
=\frac{\rho_{0}}{2}+\frac{|\varepsilon|}{\gamma} N_{0}^{2 \delta} C_{0} \rho_{0} \leq \rho_{0}
$$

for any $u \in G_{0}$, i.e., $H_{0}\left(G_{0}\right) \subset G_{0}$.

Using inequality (12), property $(P 3)$ and formula $H_{0}(u)-H_{0}\left(u^{\prime}\right)=\varepsilon L^{-1} \mathrm{P}_{N_{0}}\left(f(u)-f\left(u^{\prime}\right)\right)$ for any $u, u^{\prime} \in G_{0}$, we get the estimate

$$
\left\|H_{0}(u)-H_{0}\left(u^{\prime}\right)\right\|_{d, r} \leq \frac{|\varepsilon|}{\gamma} N_{0}^{2 \delta}\left\|f(u)-f\left(u^{\prime}\right)\right\|_{d, r} \leq C_{1} \frac{|\varepsilon|}{\gamma} N_{0}^{2 \delta}\left\|u-u^{\prime}\right\|_{d, r} .
$$

Hence, in view of formula (10) the mapping $H_{0}: \mathbf{W}^{\left(N_{0}\right)} \rightarrow \mathbf{W}^{\left(N_{0}\right)}$ is a contraction in $G_{0}$. Thus, the fixed point $u=u_{0} \in G_{0} \subset \mathbf{W}^{\left(N_{0}\right)}$ of operator $H_{0}$ is the unique solution of equation $\left(P_{N_{0}}\right)$ and

$$
B_{0} \leq 1+N_{0}^{\beta} \rho_{0}=1+\frac{|\varepsilon|}{\gamma} 2 C_{0} N_{0}^{14 \delta}
$$

Hence, the zero step of the induction is finished.

By induction, we construct the following elements of the sequence $\left\{u_{q}\right\}_{q>0}$ as $u_{q}=\sum_{i=0}^{q} h_{i}$, where $h_{0}=u_{0}$. In order to estimate their norms in the space $\mathbf{X}_{d+\beta, r+\beta}$ we will use the following lemma, whose proof will be given in the next section.

Lemma 4. The elements $u_{q}$ of sequence $\left\{u_{q}\right\}_{q \geq 0}$ satisfy the relation

$$
B_{q+1} \leq\left(1+N_{q+1}^{2 \delta}\right) B_{q} .
$$

By induction, inequality (13) yields

$$
B_{q} \leq B_{0} \prod_{i=1}^{q}\left(1+N_{i}^{2 \delta}\right)=B_{0} \prod_{i=1}^{q}\left(1+\left(N_{0}^{2^{i}}\right)^{2 \delta}\right) .
$$

Since $1+\left(N_{0}^{2^{i}}\right)^{2 \delta} \leq\left(N_{0}^{2^{i}+2^{-i}}\right)^{2 \delta}$, the following inequality holds

$$
B_{q} \leq B_{0} \prod_{i=1}^{q}\left(N_{0}^{2^{i}+2^{-i}}\right)^{2 \delta}=B_{0} N_{0}^{\sum_{i=1}^{q} 2 \delta\left(2^{i}+2^{-i}\right)}=B_{0} N_{0}^{\delta 2^{q+2}-2 \delta+\sum_{i=1}^{q} \delta 2^{1-i}} \leq B_{0} N_{q+1}^{2 \delta}
$$

Assuming that $u_{0}, u_{1}, \ldots, u_{q}$ are known, we will prove the existence of a solution $u_{q+1} \in$ $\mathbf{W}^{\left(N_{q+1}\right)}$ of the equation

$$
L u_{q+1}-\varepsilon \mathrm{P}_{N_{q+1}} f\left(u_{q+1}\right)=0 .
$$

Since $u_{q+1}=u_{q}+h_{q+1}$, we have $h_{q+1} \in \mathbf{W}^{\left(N_{q+1}\right)}$ and $B_{q+1}=1+\left\|u_{q+1}\right\|_{d+\beta, r+\beta} \leq B_{0} N_{q+2}^{2 \delta}$. Let us estimate the norm of $h_{q+1}$ in the spaces $\mathbf{X}_{d, r}$.

According to procedure of linearization of the left-hand side of equation $\left(P_{N_{q+1}}\right)$, for every $h \in \mathbf{W}^{\left(N_{q+1}\right)}$ we now write

$$
\begin{aligned}
& L\left(u_{q}+h\right)-\varepsilon \mathrm{P}_{N_{q+1}} f\left(u_{q}+h\right)=L u_{q}-\varepsilon \mathrm{P}_{N_{q+1}} f\left(u_{q}\right)+L h-\varepsilon \mathrm{P}_{N_{q+1}} \mathrm{D}_{u} f\left(u_{q}\right) h+ \\
& +\varepsilon \mathrm{P}_{N_{q+1}} \mathrm{D}_{u} f\left(u_{q}\right) h+\varepsilon \mathrm{P}_{N_{q+1}} f\left(u_{q}\right)-\varepsilon \mathrm{P}_{N_{q+1}} f\left(u_{q}+h\right)=r_{q}+\mathcal{L}_{N_{q+1}}\left(\vec{\varepsilon}, \vec{a}, u_{q}\right) h- \\
& \quad-\varepsilon \mathrm{P}_{N_{q+1}}\left(f\left(u_{q}+h\right)-f\left(u_{q}\right)-\mathrm{D}_{u} f\left(u_{q}\right) h\right)=r_{q}+\mathcal{L}_{N_{q+1}}\left(\vec{\varepsilon}, \vec{a}, u_{q}\right) h+R_{q}(h),
\end{aligned}
$$


where $r_{q}=L u_{q}-\varepsilon \mathrm{P}_{N_{q+1}} f\left(u_{q}\right), \quad R_{q}(h)=-\varepsilon \mathrm{P}_{N_{q+1}}\left(f\left(u_{q}+h\right)-f\left(u_{q}\right)-\mathrm{D}_{u} f\left(u_{q}\right) h\right)$. As a result, the element $h=h_{q+1}$ is a solution in the space $\mathbf{W}^{\left(N_{q+1}\right)}$ of the equation

$$
r_{q}+\mathcal{L}_{N_{q+1}}\left(\vec{\varepsilon}, \vec{a}, u_{q}\right) h+R_{q}(h)=0
$$

Since $u_{q}$ is a solution of equation $\left(P_{N_{q}}\right)$, i.e., $L u_{q}=\varepsilon \mathrm{P}_{N_{q}} f\left(u_{q}\right)$, we have

$$
r_{q}=\varepsilon\left(\mathrm{P}_{N_{q}}-\mathrm{P}_{N_{q+1}}\right) f\left(u_{q}\right)=-\varepsilon \mathrm{P}_{N_{q}}^{\perp} \mathrm{P}_{N_{q+1}} f\left(u_{q}\right) \in \mathbf{W}^{\left(N_{q}\right) \perp} \cap \mathbf{W}^{\left(N_{q+1}\right)} .
$$

Using formula (6) and property (P2), we obtain

$$
\left\|r_{q}\right\|_{d, r}=|\varepsilon| N_{q}^{-2 \beta}\left\|\mathrm{P}_{N_{q+1}} f\left(u_{q}\right)\right\|_{d+\beta, r+\beta} \leq|\varepsilon| C_{0} N_{q}^{-2 \beta} B_{q} \leq|\varepsilon| C_{0} B_{0} N_{q}^{-2 \beta} N_{q+1}^{2 \delta} .
$$

In order to estimate the norm $\left\|R_{q}(h)\right\|_{d, r}$ of $R_{q}(h) \in \mathbf{W}^{\left(N_{q+1}\right)}$, we use property $(P 4)$ and get

$$
\left\|R_{q}(h)\right\|_{d, r} \leq 2|\varepsilon| C_{2}\|h\|_{d, r}^{2} .
$$

Since

$$
\begin{aligned}
& R_{q}(h)-R_{q}\left(h^{\prime}\right)=-\varepsilon \mathrm{P}_{N_{q+1}}\left(f\left(u_{q}+h\right)-f\left(u_{q}+h^{\prime}\right)-\mathrm{D}_{u} f\left(u_{q}\right)\left(h-h^{\prime}\right)\right)= \\
& =-\varepsilon \mathrm{P}_{N_{q+1}}\left(f\left(u_{q}+h^{\prime}+\left(h-h^{\prime}\right)\right)-f\left(u_{q}+h^{\prime}\right)-\mathrm{D}_{u} f\left(u_{q}\right)\left(h-h^{\prime}\right)\right)
\end{aligned}
$$

for any functions $h, h^{\prime} \in \mathbf{W}^{\left(N_{q+1}\right)}$, the following estimate holds

$$
\begin{gathered}
\left\|R_{q}(h)-R_{q}\left(h^{\prime}\right)\right\|_{d, r} \leq|\varepsilon|\left\|f\left(u_{q}+h^{\prime}+\left(h-h^{\prime}\right)\right)-f\left(u_{q}+h^{\prime}\right)-\mathrm{D}_{u} f\left(u_{q}\right)\left(h-h^{\prime}\right)\right\|_{d, r} \leq \\
\leq|\varepsilon| \| f\left(u_{q}+h^{\prime}+\left(h-h^{\prime}\right)\right)-f\left(u_{q}+h^{\prime}\right)-\mathrm{D}_{u} f\left(u_{q}+h^{\prime}\right)\left(h-h^{\prime}\right)+\mathrm{D}_{u} f\left(u_{q}+h^{\prime}\right)\left(h-h^{\prime}\right)- \\
-\mathrm{D}_{u} f\left(u_{q}\right)\left(h-h^{\prime}\right) \|_{d, r} \leq|\varepsilon|\left(2 C_{2}\left\|h-h^{\prime}\right\|_{d, r}^{2}+C_{1}\left\|h-h^{\prime}\right\|_{d, r}\left\|h^{\prime}\right\|_{d, r}\right) \leq \\
\leq|\varepsilon|\left(2 C_{2}\left\|h-h^{\prime}\right\|_{d, r}\left(\|h\|_{d, r}+\left\|h^{\prime}\right\|_{d, r}\right)+C_{1}\left\|h-h^{\prime}\right\|_{d, r}\left\|h^{\prime}\right\|_{d, r}\right) \leq \\
\leq C_{3}|\varepsilon|\left(\|h\|_{d, r}+2\left\|h^{\prime}\right\|_{d, r}\right)\left\|h-h^{\prime}\right\|_{d, r},
\end{gathered}
$$

by virtue of property $(P 4)$.

By property $(P 5)$ the operator $\mathcal{L}_{N_{q+1}}\left(\vec{\varepsilon}, \vec{a}, u_{q}\right)$ is invertible for vectors $(\vec{\varepsilon}, \vec{a}) \in \mathcal{G}_{q+1}$, $u_{q} \in \mathbf{W}^{\left(N_{q}\right)}$ and $\left\|\mathcal{L}_{N_{q+1}}^{-1}\left(\vec{\varepsilon}, \vec{a}, u_{q}\right)[h]\right\|_{d, r} \leq \frac{2}{\gamma} N_{q+1}^{2 \delta}\|h\|_{d, r}$. We denote the value of the operator $H_{q+1}: \mathbf{W}^{\left(N_{q+1}\right)} \rightarrow \mathbf{W}^{\left(N_{q+1}\right)}$ on the element $h \in \mathbf{W}^{\left(N_{q+1}\right)}$ by

$$
H_{q+1}(h)=-\mathcal{L}_{N_{q+1}}^{-1}\left(\vec{\varepsilon}, \vec{a}, u_{q}\right)\left(r_{q}+R_{q}(h)\right) .
$$

Then a solution of equation $\left(\mathrm{P}_{N_{q+1}}\right)$ is a point $h=h_{q+1} \in \mathbf{W}^{\left(N_{q+1}\right)}$ of operator $H_{q+1}$, i.e., a solution of the equation $h=H_{q+1}(h)$.

We now formulate lemma which will be proved in Section 4.

Lemma 5. For every vector $(\vec{\varepsilon}, \vec{a}) \in \mathcal{G}_{q+1}$ and $q \geq 0$, the operator $H_{q+1}$ is a contraction mapping in domain

$$
G_{q+1}=\left\{h \in \mathbf{W}^{\left(N_{q+1}\right)}:\|h\|_{d, r} \leq \rho_{q+1}=4 C_{3} B_{0} \frac{|\varepsilon|}{\gamma} N_{q+1}^{-2 \delta}\right\}
$$


This lemma yields the existence of the unique solution $h_{q+1} \in \mathbf{W}^{\left(N_{q+1}\right)}$ of the equation $h=H_{q+1}(h)$ for all $(\vec{\varepsilon}, \vec{a}) \in \mathcal{G}_{q+1}$, that satisfies the relation $\left\|h_{q+1}\right\|_{d, r} \leq \rho_{q+1}=4 C_{3} B_{0} \frac{|\varepsilon|}{\gamma} N_{q+1}^{-2 \delta}$. Thus, the function $u_{q+1}=u_{q}+h_{q+1}$ is a solution of equation $\left(P_{N_{q+1}}\right)$ in the space $\mathbf{W}^{\left(N_{q+1}\right)}$ defined for all vectors $(\vec{\varepsilon}, \vec{a}) \in \mathcal{G}_{q+1} \subseteq \mathcal{G}_{0}$ and $u_{q+1}=\sum_{i=0}^{q+1} h_{i}$, where $h_{i} \in \mathbf{W}^{\left(N_{i}\right)}$.

Moreover, $\left\|h_{i}\right\|_{d, r} \leq 4 C_{3} B_{0} \frac{|\varepsilon|}{\gamma} N_{i}^{-2 \delta}$ for any $i=0,1, \ldots, q+1$.

Let us find the measure of set $\mathcal{G}_{\infty}$, whose elements satisfy Theorem 1 for any $q \geq 0$.

Theorem 2. The set $\mathcal{G}_{\infty}$ is defined by the formula $\mathcal{G}_{\infty}=\bigcap_{q \geq 0} \mathcal{G}_{q}$. Its Lebesgue measure satisfies the estimate

$$
\text { meas } \mathcal{G}_{\infty} \geq \varepsilon_{0}^{2} A^{2 p+2} \pi^{p+2}\left(1-\gamma^{2} \frac{\tilde{C} p^{2 n} \zeta_{1}(2 \kappa) \zeta_{2}(2 \alpha)}{A^{2}}\right), \quad \tilde{C}=2^{n} / \min \left\{1, C^{2 n}\right\}>0 .
$$

Proof. Since $\mathcal{G}_{q}=\bigcap_{l=0}^{q} \mathcal{G}_{l}$, we have $\mathcal{G}_{\infty}=\lim _{q \rightarrow \infty} \mathcal{G}_{q}=\bigcap_{q=0}^{\infty} \mathcal{G}_{q}=\bigcap_{q \geq 0} \mathcal{G}_{q}$. Note that

$$
\text { meas } \mathcal{G}_{\infty}=\varepsilon_{0}^{2} A^{2 p+2} \pi^{p+2}-\text { meas } \overline{\mathcal{G}_{\infty}}, \quad \overline{\mathcal{G}_{\infty}}=\bigcup_{q=0}^{\infty} \overline{\mathcal{G}_{q}}=\bigcup_{q=0}^{\infty}\left(\mathcal{O}_{\varepsilon_{0}} \times \overline{G_{q}}\right),
$$

where meas $\overline{\mathcal{G}_{\infty}}=\lim _{q \rightarrow \infty}$ meas $\overline{\mathcal{G}_{q}}$, and the bar stand for the complement of a set in the set $\mathcal{O}_{\varepsilon_{0}} \times \mathcal{O}_{A}^{p+1}$ or $\mathcal{O}_{A}^{p+1}$. Let us find the measure of the set $\overline{\mathcal{G}_{\infty}}$.

To this end, we now estimate the measure of the set $\overline{G_{q}}=\bigcup_{(k, m) \in \Omega^{N_{q}}} \overline{G_{q}(k, m)}$, where $\overline{G_{q}(k, m)}$ is the set of vectors $\vec{a}$ such that the relation

$$
\left|\lambda_{k, m}\right|<\gamma\left(1+\left\|\alpha_{k}\right\|^{2}\right)^{-\kappa_{j}}\left(1+m^{2}\right)^{-\alpha_{j}}, \quad j=1,2,
$$

is satisfied at the fixed vector $(k, m) \in \Omega^{N_{q}}$.

Consider the case $j=1$, i.e., $(k, m) \in \Omega_{1}$. Then $|\tau(m)|<\left\|\alpha_{k}\right\|$ and

$$
\left|\lambda_{k, m}\right|=\left|\alpha_{\eta k}\right|^{n}\left|a_{\hat{s}(\eta)}+\lambda_{k, m}^{*} / \alpha_{\eta k}^{n}\right|<\gamma\left(1+\left\|\alpha_{k}\right\|^{2}\right)^{-\kappa_{1}}\left(1+m^{2}\right)^{-\alpha_{1}}
$$

where $\eta$ is the minimal number in equality $\left|\alpha_{\eta k}\right|=\max _{j=1, \ldots, p}\left|\alpha_{j k}\right|, \quad \lambda_{k, m}^{*}=\lambda_{k, m}-a_{\hat{s}(\eta)} \alpha_{\eta k}^{n}$. Hence,

$$
\left|a_{\hat{s}(\eta)}+\lambda_{k, m}^{*} / \alpha_{\eta k}^{n}\right|<\frac{\gamma}{\left|\alpha_{\eta k}\right|^{n}}\left(1+\left\|\alpha_{k}\right\|^{2}\right)^{-\kappa_{1}}\left(1+m^{2}\right)^{-\alpha_{1}} .
$$

Since $p\left|\alpha_{\eta k}\right|^{2} \geq\left\|\alpha_{k}\right\|^{2} \geq C^{2}$, we have $\left|\alpha_{\eta k}\right|^{n} \geq p^{-n / 2}\left\|\alpha_{k}\right\|^{n}$. The inequality

$$
\left\|\alpha_{k}\right\|^{2} \geq \frac{C^{2}+\left\|\alpha_{k}\right\|^{2}}{2} \geq \frac{\min \left\{1, C^{2}\right\}}{2}\left(1+\left\|\alpha_{k}\right\|^{2}\right),
$$

implies $\left\|\alpha_{k}\right\|^{n} \geq\left(\frac{\min \left\{1, C^{2}\right\}}{2}\right)^{n / 2}\left(1+\left\|\alpha_{k}\right\|^{2}\right)^{n / 2}, C>0$. Thus, we have $\left|a_{\hat{s}(\eta)}+\lambda_{k, m}^{*} / \alpha_{\eta k}^{n}\right|<r_{k, m}^{*}$, where

$$
r_{k, m}^{*}=\gamma p^{n / 2}\left(\frac{\min \left\{1, C^{2}\right\}}{2}\right)^{-n / 2}\left(1+\left\|\alpha_{k}\right\|^{2}\right)^{-\kappa_{1}-n / 2}\left(1+m^{2}\right)^{-\alpha_{1}}
$$


The set of numbers $a_{\hat{s}(\eta)} \in \mathcal{O}_{A}$, satisfying this inequality, belongs to a disk of radius $r_{k, m}^{*}$. So its measure does not exceed $\pi\left(r_{k, m}^{*}\right)^{2}$. For the measure meas $\overline{G_{q}(k, m)}$ of the set $\overline{G_{q}(k, m)}$ for $(k, m) \in \Omega_{1}$ the estimate

$$
\text { meas } \overline{G_{q}(k, m)} \leq \pi^{p+1} A^{2 p} \gamma^{2} p^{n}\left(\frac{2}{\min \left\{1, C^{2}\right\}}\right)^{n}\left(1+\left\|\alpha_{k}\right\|^{2}\right)^{-2 \kappa_{1}-n}\left(1+m^{2}\right)^{-2 \alpha_{1}}
$$

hold.

Consider the case $j=2$, i.e., $(k, m) \in \Omega_{2}$. Then $|\tau(m)| \geq\left\|\alpha_{k}\right\|$ and

$$
\left|\lambda_{k, m}\right|=|\tau(m)|^{n}\left|a_{\hat{s}(0)}+\sum_{\hat{s} \neq \hat{s}(0)} a_{\hat{s}} \tau^{s_{0}}(m) \alpha_{k}^{s}\right|<\gamma\left(1+\left\|\alpha_{k}\right\|^{2}\right)^{-\kappa_{2}}\left(1+m^{2}\right)^{-\alpha_{2}} .
$$

Hence,

$$
\left|a_{\hat{s}(0)}+\sum_{\hat{s} \neq \hat{s}(0)} a_{\hat{s}} \tau^{s_{0}}(m) \alpha_{k}^{s}\right|<\frac{\gamma}{|\tau(m)|^{n}}\left(1+\left\|\alpha_{k}\right\|^{2}\right)^{-\kappa_{2}}\left(1+m^{2}\right)^{-\alpha_{2}}
$$

Since $|\tau(m)|^{2} \geq \frac{C^{2}+m^{2}}{2} \geq \frac{\min \left\{1, C^{2}\right\}}{2}\left(1+m^{2}\right)$, the following inequality

$$
|\tau(m)|^{n} \geq\left(\frac{\min \left\{1, C^{2}\right\}}{2}\right)^{n / 2}\left(1+m^{2}\right)^{n / 2}, C>0,
$$

is valid. Hence, we deduce the estimate

$$
\left|a_{\hat{s}(0)}+\sum_{\hat{s} \neq \hat{s}(0)} a_{\hat{s}} \tau^{s_{0}}(m) \alpha_{k}^{s}\right|<\gamma\left(\frac{\min \left\{1, C^{2}\right\}}{2}\right)^{-n / 2}\left(1+\left\|\alpha_{k}\right\|^{2}\right)^{-\kappa_{2}}\left(1+m^{2}\right)^{-\alpha_{2}-n / 2}
$$

For the measure meas $\overline{G_{q}(k, m)}$ of the set $\overline{G_{q}(k, m)}$ for $(k, m) \in \Omega_{2}$ the estimate

$$
\text { meas } \overline{G_{q}(k, m)} \leq \pi^{p+1} A^{2 p} \gamma^{2}\left(\frac{2}{\min \left\{1, C^{2}\right\}}\right)^{n}\left(1+\left\|\alpha_{k}\right\|^{2}\right)^{-2 \kappa_{2}}\left(1+m^{2}\right)^{-2 \alpha_{2}-n}
$$

holds.

Using the estimates (14) and (15), we get the estimate for the measure meas $\overline{G_{q}(k, m)}$ of the set $\overline{G_{q}(k, m)}$ for any vector $(k, m)$ :

$$
\text { meas } \overline{G_{q}(k, m)} \leq \tilde{C} \pi^{p+1} A^{2 p} p^{n} \gamma^{2}\left(1+\left\|\alpha_{k}\right\|^{2}\right)^{-2 \kappa}\left(1+m^{2}\right)^{-2 \alpha} .
$$

Then

$$
\begin{gathered}
\operatorname{meas} \overline{G_{q}} \leq \sum_{(k, m) \in \Omega^{N_{q}}} \operatorname{meas} \overline{G_{q}(k, m)} \leq \tilde{C} p^{2 n} \pi^{p+1} A^{2 p} \gamma^{2} \sum_{(k, m) \in \Omega^{N_{q}}}\left(1+\left\|\alpha_{k}\right\|^{2}\right)^{-2 \kappa}\left(1+m^{2}\right)^{-2 \alpha} \leq \\
\leq \tilde{C} p^{2 n} \pi^{p+1} A^{2 p} \gamma^{2} \sum_{k \in \mathbb{N}}\left(1+\left\|\alpha_{k}\right\|^{2}\right)^{-2 \kappa} \sum_{m \in \mathbb{Z}}\left(1+m^{2}\right)^{-2 \alpha} \leq \tilde{C} p^{2 n} \pi^{p+1} A^{2 p} \gamma^{2} \zeta_{1}(2 \kappa) \zeta_{2}(2 \alpha) .
\end{gathered}
$$

Since $2 \kappa>1 / 2 \beta_{0}$ and $2 \alpha>1 / 2$, we have $\zeta_{1}(2 \kappa)<\infty$ and $\zeta_{2}(2 \alpha)<\infty$. For the measure meas $\overline{\mathcal{G}_{q}}$ of the set $\overline{\mathcal{G}_{q}}$ we obtain the estimate

$$
\text { meas } \overline{\mathcal{G}_{q}} \leq \pi \varepsilon_{0}^{2} \text { meas } \overline{G_{q}} \leq \tilde{C} \pi^{p+2} \varepsilon_{0}^{2} p^{2 n} A^{2 p} \gamma^{2} \zeta_{1}(2 \kappa) \zeta_{2}(2 \alpha) .
$$


Let us find the measure meas $\overline{\mathcal{G}_{\infty}}=\lim _{q \rightarrow \infty}$ meas $\overline{\mathcal{G}_{q}}$, so

$$
\text { meas } \overline{\mathcal{G}_{\infty}} \leq \tilde{C} \pi^{p+2} \varepsilon_{0}^{2} p^{2 n} A^{2 p} \gamma^{2} \zeta_{1}(2 \kappa) \zeta_{2}(2 \alpha)
$$

The measure of the set $\mathcal{G}_{\infty}$ has the asymptotics meas $\mathcal{G}_{\infty}=\operatorname{meas}\left(\mathcal{O}_{\varepsilon_{0}} \times \mathcal{O}_{A}^{p+1}\right)+O\left(\gamma^{2}\right)$, $\gamma \rightarrow 0_{+}$, in particular,

$$
\begin{aligned}
\operatorname{meas} \mathcal{G}_{\infty} & \geq \varepsilon_{0}^{2} A^{2 p+2} \pi^{p+2}- \\
-\tilde{C} \pi^{p+2} \varepsilon_{0}^{2} p^{2 n} A^{2 p} \zeta_{1}(2 \kappa) \zeta_{2}(2 \alpha) \gamma^{2} & =\varepsilon_{0}^{2} A^{2 p+2} \pi^{p+2}\left(1-\gamma^{2} \frac{\tilde{C} p^{2 n} \zeta_{1}(2 \kappa) \zeta_{2}(2 \alpha)}{A^{2}}\right) .
\end{aligned}
$$

Theorem 3. For all vectors $(\vec{\varepsilon}, \vec{a}) \in \mathcal{G}_{\infty}$, any $\gamma>0$, and a natural number $N_{0} \geq 2$ the series $\sum_{i \geq 0} h_{i}$ converges in the space $\mathbf{X}_{d, r}$ to a solution $u$ of problem (1), (2). Its norm is determined by the inequality

$$
\|u\|_{d, r} \leq \frac{8 C_{3} B_{0}}{N_{0}^{2 \delta}} \frac{|\varepsilon|}{\gamma} .
$$

Proof. By Theorem 1, for all vectors $(\vec{\varepsilon}, \vec{a}) \in \mathcal{G}_{\infty}$ for the majorizing series $\sum_{i \geq 0}\left\|h_{i}\right\|_{d, r}$ the relation

$$
\sum_{i \geq 0}\left\|h_{i}\right\|_{d, r} \leq \sum_{i \geq 0} 4 C_{3} B_{0} \frac{|\varepsilon|}{\gamma} N_{i}^{-2 \delta}
$$

holds.

Then the series $\sum_{i \geq 0} h_{i}$ converges in the space $\mathbf{X}_{d, r}$ to some function $u \in \mathbf{X}_{d, r}$, because

$$
\|u\|_{d, r} \leq \sum_{i \geq 0}\left\|h_{i}\right\|_{d, r} \leq 4 C_{3} B_{0} \frac{|\varepsilon|}{\gamma} \sum_{i \geq 0}\left(N_{0}^{2^{i}}\right)^{-2 \delta} \leq 4 C_{3} B_{0} \frac{|\varepsilon|}{\gamma} \frac{2}{N_{0}^{2 \delta}}=\frac{8 C_{3} B_{0}}{N_{0}^{2 \delta}} \frac{|\varepsilon|}{\gamma} .
$$

We now show that $L u=\varepsilon f(u)$. The function $u_{q}$ is a solution of equation $\left(P_{N_{q}}\right)$. Then

$$
L u_{q}=\varepsilon P_{N_{q}} f\left(u_{q}\right)=\varepsilon f\left(u_{q}\right)-\varepsilon P_{N_{q}}^{\perp} f\left(u_{q}\right) .
$$

With regard for the property (6) of the projector $P_{N_{q}}^{\perp}$, property $(P 2)$ and the estimate for $B_{q}$ in the proof of Theorem 1, we have

$$
\begin{gathered}
\left\|P_{N_{q}}^{\perp} f\left(u_{q}\right)\right\|_{d, r} \leq N_{q}^{-2 \beta}\left\|f\left(u_{q}\right)\right\|_{d+\beta, r+\beta} \leq C_{0} N_{q}^{-2 \beta} B_{q} \leq C_{0} B_{0} N_{q}^{-24 \delta} N_{q+1}^{2 \delta}= \\
=C_{0} B_{0} N_{q}^{-24 \delta} N_{q}^{4 \delta}=C_{0} B_{0} N_{q}^{-20 \delta}=C_{0} B_{0} N_{0}^{-20 \delta 2^{q}} .
\end{gathered}
$$

Thus, $P_{N_{q}}^{\perp} f\left(u_{q}\right) \rightarrow 0$ as $q \rightarrow \infty$. Then, by virtue of property $(P 1)$ the right-hand side of (16) converges to $\varepsilon f(u)$ in the space $\mathbf{X}_{d, r}$. The continuity of the operator $L$ implies that the left-hand side of (16) $L u_{q}$ converges to $L u$ as $q \rightarrow \infty$ in the sense of distributions.

\section{Proof of auxiliary lemmas.}

Proof of Lemma 1. For all vectors $\vec{a} \in G_{q}$ the operator $|\mathcal{D}|^{-1 / 2}$ exists. Since

$$
h=\sum_{(k, m) \in \Omega^{N_{q}}} e^{\tau(m) t} x_{k} h_{k, m}
$$


we have

$$
|\mathcal{D}|^{-1 / 2} h=\sum_{(k, m) \in \Omega^{N_{q}}} \frac{h_{k, m} \varphi_{k, m}}{\sqrt{\left|\lambda_{k, m}\right|}} .
$$

Moreover, inequality (9) yields the estimate

$$
\begin{gathered}
\left\||\mathcal{D}|^{-1 / 2} h\right\|_{d^{*}, r^{*}, \Omega_{j}}^{2}=\sum_{(k, m) \in \Omega^{N_{q}}}\left(1+\left\|\alpha_{k}\right\|^{2}\right)^{d^{*}}\left(1+m^{2}\right)^{r^{*}} \frac{\left|h_{k, m}\right|^{2}}{\left|\lambda_{k, m}\right|} \leq \\
\leq \sum_{(k, m) \in \Omega^{N_{q}}} \frac{1}{\gamma}\left(1+\left\|\alpha_{k}\right\|^{2}\right)^{d^{*}+\kappa_{j}}\left(1+m^{2}\right)^{r^{*}+\alpha_{j}}\left|h_{k, m}\right|^{2}=\frac{1}{\gamma}\|h\|_{d^{*}+\kappa_{j}, r^{*}+\alpha_{j}, \Omega_{j}}^{2} .
\end{gathered}
$$

Proof of Lemma 2. Since $\mathcal{U}=|\mathcal{D}|^{-1 / 2} \mathcal{D}|\mathcal{D}|^{-1 / 2}$, its action on function $h \in \mathbf{X}_{d^{*}, r^{*}}$ is given by the formula

$$
\mathcal{U} h=\sum_{(k, m) \in \mathbb{N} \times \mathbb{Z}} \frac{\lambda_{k, m}}{\left|\lambda_{k, m}\right|} h_{k, m} \varphi_{k, m},
$$

where $\lambda_{k, m}$ are eigenvalues of the operator $\mathcal{D}$. Using the definitions of the space $\mathbf{X}_{d^{*}, r^{*}}$ and the norm in this space, we obtain $\left\|\mathcal{U}^{-1} h\right\|_{d^{*}, r^{*}}=\|h\|_{d^{*}, r^{*}}$.

Proof of Lemma 3. We note that $\mathcal{R}_{1} h=\varepsilon|\mathcal{D}|^{-1 / 2} \mathrm{P}_{N_{q}}\left(D_{u} f|\mathcal{D}|^{-1 / 2} h\right)$. Then, in view of the Lemma 1, property (P3) and inequalities (5), we obtain

$$
\begin{aligned}
& \left\|\mathcal{R}_{1} h\right\|_{\bar{d}, \bar{r}, \Omega_{j}} \leq \frac{|\varepsilon|}{\sqrt{\gamma}}\left\|\mathrm{P}_{N_{q}} \mathrm{D}_{u} f|\mathcal{D}|^{-1 / 2} h\right\|_{\bar{d}+\kappa_{j}, \bar{r}+\alpha_{j}, \Omega_{j}} \leq \frac{|\varepsilon|}{\sqrt{\gamma}} N_{q}^{-\delta}\left\|\mathrm{D}_{u} f|\mathcal{D}|^{-1 / 2} h\right\|_{\bar{d}+2 \kappa_{j}, \bar{r}+2 \alpha_{j}, \Omega_{j}} \leq \\
& \leq \frac{|\varepsilon|}{\sqrt{\gamma}} N_{q}^{-\delta} C_{1}\left\||\mathcal{D}|^{-1 / 2} h\right\|_{\bar{d}, \bar{r}, \Omega_{j}} \leq C_{1} \frac{|\varepsilon|}{\gamma} N_{q}^{-\delta}\|h\|_{\bar{d}+\kappa_{j}, \bar{r}+\alpha_{j}, \Omega_{j}} \leq C_{1} \frac{|\varepsilon|}{\gamma}\|h\|_{\bar{d}, \bar{r}, \Omega_{j}} \leq C_{1} \frac{|\varepsilon|}{\gamma}\|h\|_{\bar{d}, \bar{r}},
\end{aligned}
$$

$j=1,2$. So, $\left\|\mathcal{R}_{1} h\right\|_{\bar{d}, \bar{r}} \leq C_{1} \frac{|\varepsilon|}{\gamma}\|h\|_{\bar{d}, \bar{r}}$.

Proof of Lemma 4. Since $u_{q+1}=u_{q}+h_{q+1}$ i $h_{q+1} \in G_{q+1}$, we have

$$
B_{q+1}=1+\left\|u_{q+1}\right\|_{d+\beta, r+\beta} \leq 1+\left\|u_{q}\right\|_{d+\beta, r+\beta}+\left\|h_{q+1}\right\|_{d+\beta, r+\beta}=B_{q}+\left\|h_{q+1}\right\|_{d+\beta, r+\beta} .
$$

The norm of the solution $h_{q+1}$ of the equation $h_{q+1}=-\mathcal{L}_{N_{q+1}}^{-1}\left(\vec{\varepsilon}, \vec{a}, u_{q}\right)\left(r_{q}+R_{q}\left(h_{q+1}\right)\right)$ in the space $\mathbf{X}_{d+\beta, r+\beta}$ satisfies the estimate

$$
\left\|h_{q+1}\right\|_{d+\beta, r+\beta} \leq \frac{2}{\gamma} N_{q+1}^{2 \delta}\left(\left\|r_{q}\right\|_{d+\beta, r+\beta}+\left\|R_{q}\left(h_{q+1}\right)\right\|_{d+\beta, r+\beta}\right) .
$$

We note that $r_{q}=-\varepsilon \mathrm{P}_{N_{q}}^{\perp} \mathrm{P}_{N_{q+1}} f\left(u_{q}\right)$. Therefore, we can write, by using $(P 2)$, the estimate for the norm $\left\|r_{q}\right\|_{d+\beta, r+\beta}$ as follows:

$$
\left\|r_{q}\right\|_{d+\beta, r+\beta} \leq|\varepsilon|\left\|f\left(u_{q}\right)\right\|_{d+\beta, r+\beta} \leq|\varepsilon| C_{0}\left(1+\left\|u_{q}\right\|_{d+\beta, r+\beta}\right)=|\varepsilon| C_{0} B_{q} .
$$

To estimate the quantities $\left\|R_{q}\left(h_{q+1}\right)\right\|_{d+\beta, r+\beta}$ we use Lemma 2 and property $(P 4)$ and get

$$
\left\|R_{q}\left(h_{q+1}\right)\right\|_{d+\beta, r+\beta} \leq|\varepsilon| C_{2}\left(\left\|u_{q}\right\|_{d+\beta, r+\beta}\left\|h_{q+1}\right\|_{d, r}^{2}+\left\|h_{q+1}\right\|_{d, r}\left\|h_{q+1}\right\|_{d+\beta, r+\beta}\right) \leq
$$




$$
\leq|\varepsilon| C_{2}\left(B_{q} \rho_{q+1}^{2}+\rho_{q+1}\left\|h_{q+1}\right\|_{d+\beta, r+\beta}\right) .
$$

Substituting estimates (18) and (19) in inequality (17), we have

$$
\begin{gathered}
\left\|h_{q+1}\right\|_{d+\beta, r+\beta} \leq \frac{2}{\gamma} N_{q+1}^{2 \delta}\left(|\varepsilon| C_{0} B_{q}+|\varepsilon| C_{2}\left(B_{q} \rho_{q+1}^{2}+\rho_{q+1}\left\|h_{q+1}\right\|_{d+\beta, r+\beta}\right)\right)= \\
=2 C_{0} \frac{|\varepsilon|}{\gamma} N_{q+1}^{2 \delta} B_{q}+2 C_{2} \frac{|\varepsilon|}{\gamma} N_{q+1}^{2 \delta} \rho_{q+1}^{2} B_{q}+2 C_{2} \frac{|\varepsilon|}{\gamma} N_{q+1}^{2 \delta} \rho_{q+1}\left\|h_{q+1}\right\|_{d+\beta, r+\beta}< \\
<2 C_{3} \frac{|\varepsilon|}{\gamma} N_{q+1}^{2 \delta} B_{q}+C_{3} \frac{|\varepsilon|}{\gamma} N_{q+1}^{2 \delta} \rho_{q+1}^{2} B_{q}+C_{3} \frac{|\varepsilon|}{\gamma} N_{q+1}^{2 \delta} \rho_{q+1}\left\|h_{q+1}\right\|_{d+\beta, r+\beta} .
\end{gathered}
$$

In view of Lemma 5 and equality (10) for $|\varepsilon|<\varepsilon_{0}$, from the inequality

$$
4 C_{3} \frac{|\varepsilon|}{\gamma} N_{q+1}^{2 \delta} \rho_{q+1}<4 C_{3} \frac{\varepsilon_{0}}{\gamma} N_{q+1}^{2 \delta} \rho_{q+1} \leq 4\left(\frac{2 C_{3} \varepsilon_{0}}{\gamma}\right)^{2}=\frac{2}{3}<1
$$

we obtain the following estimate for $q \geq 2$

$$
\begin{gathered}
\left\|h_{q+1}\right\|_{d+\beta, r+\beta} \leq 2 C_{3} \frac{|\varepsilon|}{\gamma} N_{q+1}^{2 \delta} B_{q}+\frac{\rho_{q+1}}{4} B_{q}+\frac{1}{4}\left\|h_{q+1}\right\|_{d+\beta, r+\beta}< \\
<2 C_{3} \frac{|\varepsilon|}{\gamma} N_{q+1}^{2 \delta} B_{q}+C_{3} B_{0} \frac{|\varepsilon|}{\gamma} N_{q+1}^{-2 \delta} B_{q}+\frac{1}{4}\left\|h_{q+1}\right\|_{d+\beta, r+\beta}= \\
=2 C_{3} \frac{|\varepsilon|}{\gamma} B_{q}\left(N_{q+1}^{2 \delta}+\frac{1}{2} B_{0} N_{q+1}^{-2 \delta}\right)+\frac{1}{4}\left\|h_{q+1}\right\|_{d+\beta, r+\beta} \leq 4 C_{3} \frac{|\varepsilon|}{\gamma} N_{q+1}^{2 \delta} B_{q}+\frac{1}{4}\left\|h_{q+1}\right\|_{d+\beta, r+\beta} .
\end{gathered}
$$
yield

Then $\frac{3}{4}\left\|h_{q+1}\right\|_{d+\beta, r+\beta} \leq 4 C_{3} \frac{|\varepsilon|}{\gamma} N_{q+1}^{2 \delta} B_{q}$ for $q \geq 2$. This result and relation (10) for $|\varepsilon|<\varepsilon_{0}$

$$
\begin{gathered}
\left\|h_{q+1}\right\|_{d+\beta, r+\beta} \leq \frac{16}{3} C_{3} \frac{|\varepsilon|}{\gamma} N_{q+1}^{2 \delta} B_{q} \leq N_{q+1}^{2 \delta} B_{q}, \quad q \geq 2, \\
\left\|h_{1}\right\|_{d+\beta, r+\beta} \leq \frac{8}{3} C_{3} \frac{|\varepsilon|}{\gamma}\left(1+\alpha_{1}\right) N_{1}^{2 \delta} B_{0}, \quad\left\|h_{2}\right\|_{d+\beta, r+\beta} \leq \frac{8}{3} C_{3} \frac{|\varepsilon|}{\gamma}\left(1+\alpha_{2}\right) N_{2}^{2 \delta} B_{1},
\end{gathered}
$$

where $\alpha_{1}=\frac{1}{2} B_{0} N_{0}^{-8 \delta}, \alpha_{2}=\frac{1}{2} B_{0} N_{0}^{-16 \delta}$.

From (10) we have $|\varepsilon|<y_{2}$, so

$$
|\varepsilon|<\frac{3 \gamma}{8 C_{3}\left(1+\frac{1}{2} N_{0}^{-8 \delta}\left(1+\frac{|\varepsilon|}{\gamma} 2 C_{0} N_{0}^{14 \delta}\right)\right)} .
$$

Moreover, $|\varepsilon|<\frac{3 \gamma}{8 C_{3}\left(1+\alpha_{1}\right)}$. Then $\left\|h_{1}\right\|_{d+\beta, r+\beta} \leq N_{1}^{2 \delta} B_{0}$.

Similarly, using the formula (10), we have $|\varepsilon|<y_{2}$, so

$$
|\varepsilon|<\frac{3 \gamma}{8 C_{3}\left(1+\frac{1}{2} N_{0}^{-16 \delta}\left(1+\frac{|\varepsilon|}{\gamma} 2 C_{0} N_{0}^{14 \delta}\right)\right)},
$$

then $|\varepsilon|<\frac{3 \gamma}{8 C_{3}\left(1+\alpha_{2}\right)}$ and $\left\|h_{2}\right\|_{d+\beta, r+\beta} \leq N_{2}^{2 \delta} B_{1}$. Hence, $B_{q+1} \leq B_{q}+N_{q+1}^{2 \delta} B_{q}=\left(1+N_{q+1}^{2 \delta}\right) B_{q}$, which finishes the proof. 
Proof of Lemma 5. For any $h \in G_{q+1}$ with regard for property (P5), we have

$$
\left\|H_{q+1}(h)\right\|_{d, r} \leq \frac{2}{\gamma} N_{q+1}^{2 \delta}\left(\left\|r_{q}\right\|_{d, r}+\left\|R_{q}(h)\right\|_{d, r}\right) \leq 2 C_{0} B_{0} \frac{|\varepsilon|}{\gamma} N_{q+1}^{2 \delta} N_{q}^{-2 \beta} N_{q+1}^{2 \delta}+4 C_{2} \frac{|\varepsilon|}{\gamma} N_{q+1}^{2 \delta}\|h\|_{d, r}^{2} .
$$

In view of the value of $\beta$, equality $N_{q+1}=N_{q}^{2}$, and formula (10) and $|\varepsilon|<\varepsilon_{0}$, we can write the inequality

$$
\begin{gathered}
\left\|H_{q+1}(h)\right\|_{d, r} \leq 2 C_{0} B_{0} \frac{|\varepsilon|}{\gamma} N_{q+1}^{-2 \delta}+4 C_{2} \frac{|\varepsilon|}{\gamma} N_{q+1}^{2 \delta} \rho_{q+1}^{2} \leq \\
\leq 2 C_{3} B_{0} \frac{|\varepsilon|}{\gamma} N_{q+1}^{-2 \delta}+2 C_{3} \frac{|\varepsilon|}{\gamma} N_{q+1}^{2 \delta} \rho_{q+1}^{2} \leq \frac{\rho_{q+1}}{2}+8 B_{0}\left(C_{3} \frac{|\varepsilon|}{\gamma}\right)^{2} \rho_{q+1} \leq \rho_{q+1} .
\end{gathered}
$$

Therefore, the operator $H_{q+1}$ maps $G_{q+1}$ into itself.

Any functions $h, h^{\prime} \in G_{q+1}$ satisfy the equality

$$
H_{q+1}(h)-H_{q+1}\left(h^{\prime}\right)=-\mathcal{L}_{N_{q+1}}^{-1}\left(\vec{\varepsilon}, \vec{a}, u_{q}\right)\left(R_{q}(h)-R_{q}\left(h^{\prime}\right)\right),
$$

then we can write the estimate

$$
\begin{gathered}
\left\|H_{q+1}(h)-H_{q+1}\left(h^{\prime}\right)\right\|_{d, r} \leq \frac{2}{\gamma} N_{q+1}^{2 \delta}\left\|R_{q}(h)-R_{q}\left(h^{\prime}\right)\right\|_{d, r} \leq \\
\leq 2 C_{3} \frac{|\varepsilon|}{\gamma} N_{q+1}^{2 \delta}\left(\|h\|_{d, r}+2\left\|h^{\prime}\right\|_{d, r}\right)\left\|h-h^{\prime}\right\|_{d, r} \leq \\
\leq 2 C_{3} \frac{|\varepsilon|}{\gamma} N_{q+1}^{2 \delta} 12 C_{3} B_{0} \frac{|\varepsilon|}{\gamma} N_{q+1}^{-2 \delta}\left\|h-h^{\prime}\right\|_{d, r} \leq 24 B_{0}\left(C_{3} \frac{|\varepsilon|}{\gamma}\right)^{2}\left\|h-h^{\prime}\right\|_{d, r} .
\end{gathered}
$$

Formula (10) implies that $|\varepsilon|<y_{1}$ and

$$
|\varepsilon|<\frac{\gamma}{2 \sqrt{6} C_{3} \sqrt{1+\frac{|\varepsilon|}{\gamma} 2 C_{0} N_{0}^{14 \delta}}} .
$$

Hence, $|\varepsilon|<\frac{\gamma}{2 C_{3} \sqrt{6 B_{0}}}$. Thus, the operator $H_{q+1}$ is contractive.

\section{REFERENCES}

1. V.I. Arnol'd, Small denominators. I. Mapping the circle onto itself, Izv. Akad. Nauk SSSR Ser. Mat., 25, 1961, P. 21-86. (in Russian)

2. M. Berti, P. Bolle, Cantor families of periodic solutions for completely resonant nonlinear wave equations, Duke Math. J., 134 (2006), №2, 359-419.

3. M. Berti, P. Bolle, Cantor families of periodic solutions of wave equations with $C^{k}$ nonlinearities, Nonlinear Differential Equations and Applications, 15 (2008), 247-276.

4. M. Berti, P. Bolle, Sobolev periodic solutions of nonlinear wave equations in higher spatial dimensions, Arch. Rational Mech. Anal., 195 (2010), №2, 609-642.

5. V.S. Il'kiv, Incorrect nonlocal boundary value problem for partial differential equations, North-Holland Mathematics Studies, 197(C) (2004), 115-121.

6. V.S. Il'kiv, Z.M. Nytrebych, P.Y. Pukach, Boundary-value problems with integral conditions for a system of Lame equations in the space of almost periodic functions, Electronic Journal of Differential Equations, 2016 (2106), №304, 1-12. 
7. V.S. Il'kiv, N.I. Strap, Nonlocal boundary value problem for a differential-operator equation with nonlinear right part in a complex domain, Mat. Stud., 45 (2016), №2, 170-181. (in Ukrainian)

8. V.S. Il'kiv, N.I. Strap, Nonlocal boundary value problem for a differential-operator equation with nonlinearity in the spaces of Dirichlet-Taylor series with fixed spectrum, Mathematical Methods and Physicomechanical Fields, 59 (2016), №2, 77-85. (in Ukrainian)

9. V.S. Il'kiv, N.I. Strap, Solvability of a nonlocal boundary-value problem for the operator-differential equation with weak nonlinearity in a refined scale of Sobolev spaces, Journal of Mathematical Sciences, 218 (2016), №1, 1-15.

10. V.S. Il'kiv, N.I. Strap, Solvability of the nonlocal boundary-value problem for a system of differentialoperator equations in the Sobolev scale of spaces and in a refined scale, Ukrainian Mathematical Journal, 67 (2015), №5, 690-710.

11. P.I. Kalenyuk, I.V. Kohut, Z.M. Nytrebych, Problem with nonlocal two-point condition in time for a homogeneous partial differential equation of infinite order with respect to space variables, Journal of Mathematical Sciences, 167 (2010), №1, 1-15.

12. O. Malanchuk, Z. Nytrebych, Homogeneous two-point problem for PDE of the second order in time variable and infinite order in spatial variables, Open Mathematics, 15 (2017), №1, 101-110.

13. J. Mozer, A rapidly convergent iteration method and non-linear differential equations I, Annali della Scuola Norm. Super. de Piza ser. III, 20(1966), №2, 265-315.

14. J. Mozer, A rapidly convergent iteration method and non-linear differential equations II, Annali della Scuola Norm. Super. de Piza ser. III, 20, №3, (1966), 499-535.

15. Ptashnyk B.Yo., Il'kiv V.S., Kmit' I.Ya., Polishchuk V.M., Nonlocal boundary value problems for partial differential equations, Naukova Dumka, Kyiv, 2002, 415 p. (in Ukrainian)

Lviv Polytechnic National University, Lviv, Ukraine, i.volyanska@i.ua

Lviv Polytechnic National University, Lviv, Ukraine, ilkivv@i.ua

College of Oil and Gas, Drohobych, Lviv region, Ukraine, n.strap@i.ua 